EXPERIMENTAL STUDY

\title{
Clinical and molecular evidence for DAX-1 inhibition of steroidogenic factor-1-dependent ACTH receptor gene expression
}

\author{
Oliver Zwermann, Felix Beuschlein, Enzo Lalli ${ }^{3}$, Albrecht Klink, Paolo Sassone-Corsi ${ }^{1}$ and Martin Reincke $^{2}$ \\ Division of Endocrinology, Department of Internal Medicine II, University of Freiburg, Germany, ${ }^{1}$ Institut de Génétique et Biologie Moléculaire et \\ Cellulaire, CNRS, INSERM, Université Louis Pasteur Illkirch-CU de Strasbourg, France, ${ }^{2}$ Medizinische Klinik-Innenstadt Ziemssenstr. 1 , D-80336 \\ Munich, Germany and ${ }^{3}$ Institut de Pharmacologie Moleculaire et Cellulaire, CNRS UMR 6097, Valbonne, France
}

(Correspondence should be addressed to M Reincke; Email: martin.reincke@med.uni-muenchen.de)

\begin{abstract}
Background: The ACTH receptor (ACTH-R) is a member of the seven transmembrane domain receptor super-family. In non-functional adrenal adenomas and adrenocortical carcinomas, ACTH-R expression is low. However, no inhibitory factor for ACTH-R expression has been defined to date. DAX-1 (dosage-sensitive sex reversal, adrenal hypoplasia congenita, critical region on the $\mathrm{X}$ chromosome, gene-1) is a general repressor of steroid production, inhibiting steroidogenic factor-1 (SF-1)-dependent expression of multiple steroidogenic enzymes. The aim of this study was to investigate whether ACTH-R gene transcription is affected by DAX-1 and whether this mechanism is involved in down-regulation of ACTH-R expression in adrenocortical tumors.

Methods: We screened 22 adrenocortical tumors for ACTH-R and DAX-1 mRNA expression by Northern blot. For in vitro analyses we co-transfected mouse Y1 adrenocortical carcinoma cells with the luciferase reporter gene vector pGL3 containing full-length constructs of human $(\mathrm{h})$ or mouse $(\mathrm{m})$ ACTH-R promoter together with a DAX-1 expression plasmid. These experiments were also performed using ACTH-R promoter 5'-deletion constructs and constructs mutated at the SF-1-binding sites. Results: We found a negative correlation between DAX-1 and ACTH-R mRNA expression $(R=-0.47$, $P<0.02)$. Accordingly, in vitro expression of DAX-1 significantly reduced hACTH-R and mACTH-R promoter activity by 89 and 55\% respectively. DAX-1 inhibition was also present in the shortest construct of a series of $5^{\prime}$-deletion constructs of the human promoter extending from -64 to $+40 \mathrm{bp}$ relative to the transcription start site. Mutation of the SF-1-binding sites within the hACTH-R promoter resulted in reduced or abolished DAX-1 inhibition, arguing for a mechanism that involves SF-1 for DAX-1 inhibition.

Conclusions: These data support the concept that DAX-1 is a major repressor of ACTH-R gene expression in vitro and in vivo.
\end{abstract}

European Journal of Endocrinology 152 769-776

\section{Introduction}

The adrenocorticotropin receptor (ACTH-R) is one of the smallest members of the seven transmembrane domain, G-protein coupled receptor super-family (1) and transmits the ACTH hormonal signal to enhance glucocorticoid, androgen and short-term mineralocorticoid production mainly via elevation of intracellular cAMP levels. Although a positive correlation between expression of P450scc (side chain cleavage enzyme) and ACTH-R has been demonstrated in adrenal tumors, it remains unclear which common transcription factors and upstream regulators modulate their expression (2). Several putative binding sites, such as activator protein-1 sites, cAMP responsive elements and steroidogenic factor-1 (SF-1)-binding sites within the ACTH-R promoter region have been described; only a few, however, have been functionally characterized so far $(3,4)$. In non-functioning adrenal adenomas and adrenocortical carcinomas ACTH-R expression is down-regulated or lost in the majority of cases $(5,6)$. Although in a subset of adrenocortical carcinomas diminished ACTH-R expression is associated with genomic loss of heterozygosity of the ACTH-R locus, other mechanisms leading to decreased ACTH-R expression must exist. To date, however, no inhibitory factors for ACTH-R expression have been described.

SF-1 (NR5A1) and DAX-1 (dosage-sensitive sex reversal, adrenal hypoplasia congenita, critical region on the $\mathrm{X}$ chromosome, gene-1, NR0B1) are two orphan nuclear receptors that have been demonstrated to contribute significantly to adrenocortical function. Whereas SF-1 has been characterized as a transcription factor required for the expression of a variety of 
steroidogenic enzymes (7), DAX-1 has been shown to serve as a general repressor of steroid biosynthesis by inhibiting the transcription of StAR (steroidogenic acute regulatory protein), $3 \beta$-hydroxysteroid dehydrogenase and $\mathrm{P} 450 \mathrm{scc}$ genes as well as its own transcription $(8,9)$. Beside its nuclear inhibitory effect on transcription of these proteins, DAX-1 also acts at the post-transcriptional level by binding to polyribosomes in the cytosol (10). Functional interplay between DAX-1 and SF-1 is complex and includes both agonistic and antagonistic interaction.

As such, SF-1 has been shown to bind and activate the DAX-1 promoter $(11,12)$. However, in vitro studies have demonstrated that DAX-1 can physically interact with SF-1 and inhibit SF-1-mediated transcription of steroidogenic enzymes (13), anti-Mullerian hormone (14) and DAX-1 itself (8), most likely by recruitment of transcriptional co-repressors (15). Alternatively, as demonstrated for the StAR promoter, DAX-1 can inhibit gene expression by binding to unique hairpin structures in the promoter region, thus blocking transcriptional activity independently from SF-1 activation (8). Since DAX-1 protein is not exclusively located within the nucleus and has been demonstrated to bind to polyadenylated RNA and polyribosomes in the cytoplasm, these findings raise the possibility that DAX-1 exerts inhibitory effects also at the post-transcriptional level (10). Taken together, there is good experimental evidence that SF-1 and DAX-1 are functional counterparts in order to maintain tissue-specific regulation of genes in steroid-producing and reproductive tissue.

Interestingly, DAX-1 expression pattern in adrenal tumors argues for an implication of DAX-1 in steroidogenesis within the context of adrenal tumorigenesis, with lowest DAX-1 expression in hormonally active tumors and highest DAX-1 expression levels in endocrine-inactive adenomas (16). Since we could also demonstrate that ACTH-R expression in adrenal tumors is highest in aldosterone- and cortisol-secreting adenomas and low in endocrine-inactive adenomas in another set of experiments (2), we hypothesized that DAX-1 might repress ACTH-R promoter activity. Indeed, herein we present evidence that DAX-1 represses the transcription of the ACTH-R gene in adrenocortical tumors by a mechanism that involves SF-1binding sites within the ACTH-R promoter.

\section{Materials and methods}

\section{Extraction of RNA from adrenocortical tumor tissue and Northern blot}

Tissues from seven normal adrenals, three non-functional adenomas, eight cortisol-producing adenomas, five aldosterone-producing adenomas, five adrenocortical carcinomas and one multinodular adrenal hyperplasia were studied. The clinical and pathological diagnosis was made according to established criteria.
Normal adrenal tissue was collected from the adjacent normal cortex of aldosterone-producing adenomas or from brain-dead patients. Neoplastic adrenal tissue was collected with the approval of the Ethical Committee of the University Hospital of Würzburg and Freiburg. After removing adjacent fat, the tissues were snap-frozen and immediately stored at $-80^{\circ} \mathrm{C}$ until processing. Poly A + RNA was isolated from approximately $0.1 \mathrm{~g}$ adrenal tissue using the Oligotex Direct mRNA Kit (Qiagen). For Northern blotting, $3 \mu \mathrm{g}$ poly $\mathrm{A}+\mathrm{RNA}$ were electrophoresed through a $1 \%$ agarose/formaldehyde gel and blotted to a nylon membrane (Qiagen) overnight. cDNA probes for human ACTH-R (hACTH-R) (primers: 5'GATTTAACTTAGATCTCCAGCAAG $^{\prime}$ and 5'ACACTATTCTGGCACTTGGCAACG3') and DAX-1 (17) were generated by PCR. For standardization the blots were hybridized with a mouse $\beta$-actin cDNA probe (Stratagene, Heidelberg, Germany). Labeling of the cDNAs with $\left[{ }^{32} \mathrm{P}\right] \mathrm{dCTP}$ was performed using a Random Primed Labeling Kit (Roche Diagnostics). The blots were washed under high stringency conditions and exposed to Kodak Xomat films at $-80^{\circ} \mathrm{C}$ with intensifying screens. The relative intensities of the major hybridization signals (hACTH-R: $1.8 \mathrm{~kb}$ transcript, DAX-1: $1.5 \mathrm{~kb}$ transcript) were quantified by scanning densitometry with a video camera and a Macintosh computer-based image analysis system using the IMAGE program (National Institutes of Health, Bethesda, MD, USA). Results were expressed as percent of normal adrenal tissue (100\%) after normalization for $\beta$-actin expression. Correlation between hACTH-R mRNA expression and DAX-1 mRNA levels was determined by linear regression analysis and expressed as Pearson's correlation coefficient. $P<0.05$ was considered statistically significant.

\section{Cell culture and transfections}

Y1 cells were cultured in Ham F10 medium supplemented with $7.5 \%$ horse serum, $2.5 \%$ fetal bovine serum, $2 \mathrm{mmol} / \mathrm{l}$ glutamine and $100 \mathrm{U} / \mathrm{ml}$ penicillin/ streptomycin. For transfections cells were grown in 24 -well plates to a density of $\sim 80 \%$. Six hundred nanograms of DNA of each reporter construct and expression vectors (pSV-DAX-1, pSV-SF1 (17)) and $400 \mathrm{ng}$ lacZ vector were transfected by ExGen 500 (MBI Fermentas, Karlsruhe, Germany) with an ExGen/DNA ratio of 6 following the instructions of the manufacturer. For basal values, empty vector pSG5 was co-transfected to keep the amount of DNA at a constant level. After $48 \mathrm{~h}$ cells were harvested in $100 \mu \mathrm{l}$ Lysis Buffer and $50 \mu \mathrm{l}$ were used for luciferase measurement using the Luciferase Reporter Gene Assay High Sensitivity (Roche Diagnostics) and a Lumat 9006 (EG\&G Berthold, Bad Wildbad, Germany) as a luminometer. Luciferase measurements were normalized for $\beta$-galactosidase using $20 \mu \mathrm{l}$ cell lysate and the Galacto-Light Kit (Tropix, Langen, Germany), 
or protein concentration, determined by the Lowry method (Sigma-Aldrich). Every transfection experiment was run in duplicate and repeated at least twice. The results are expressed as relative units in \% with the $-1017 /+40$ promoter construct set to $100 \%$. Background activity of the empty vector was in the range of $5-7 \%$ and was subtracted in all experiments.

\section{Generation of ACTH-R promoter luciferase constructs in pGL3}

A $1 \mathrm{~kb}$ fragment of the hACTH-R and mouse (m) ACTH-R promoter was amplified by PCR from a cosmid bank (RZPD library No. 111; RZPD, Heidelberg, Germany). Conditions were 30 cycles of $30 \mathrm{~s}$ at $95^{\circ} \mathrm{C}$, $45 \mathrm{~s}$ at $56^{\circ} \mathrm{C}$ and $75 \mathrm{~s}$ at $72{ }^{\circ} \mathrm{C}$. Five different primers containing a $\mathrm{KpnI}$ site at their $5^{\prime}$-end and a primer containing a BglII site were used to generate fulllength and truncated hACTH-R promoter constructs. $5^{\prime}$-primer sequences were for the $\mathrm{p}(-1017 /+40)$ fragment: 5'CGGGGTACCCTGCAGGGCATGTTGCGG3'; for $\mathrm{p}(-783 /+40): \quad 5^{\prime}$ CGGGGTACCTTCAGAAACAGCCACTGG3 $^{\prime}$; for $\mathrm{p}(-549 /+40)$ : 5'CGGGGTACCCATTGGCAGAACACAGGG3'; for $\mathrm{p}(-293 /+40)$ : 5'CGGGGTACCTAAATGCAACTCTAAAGTGG $3^{\prime}$; for $\mathrm{p}(-214 /+40)$ : 5'TCAGTGGTACCGGAAGTAACCTTGACTAGC3'; for $\mathrm{p}(-112 /+40):$ 5'CGGGGTACCGGGATGACATTTATTCAAGG3'; and for $\mathrm{p}(-64 /+40)$ : 5'TCAGTGGTACCGTGATGCATGTGTTCCGG3'. Primer sequence for the $3^{\prime}$ primer at position +40 containing a BglII site was 5'GCGCGCGCAGATCTAAGCAGGAACTTTCTGGG3'. The resulting PCR products were purified by gel electrophoresis and ligated into the luciferase reporter gene vector pGL3 (Promega) using a KpnI and a BglII restriction site. Following transformation and amplification in $\mathrm{DH} 5 \alpha$ cells, the plasmids were extracted using the Qiagen Maxi Prep Kit (Qiagen). All plasmids were sequenced to exclude mutations within the DNA sequence.

\section{Site-directed mutagenesis of promoter constructs}

Mutations replacing the two guanines in the consensus SF-1-binding site (PyCAAGGPyCPu) (18) by two adenines (19) at each of the three SF-1-binding sites at position $-35,-98$ and -209 of the hACTH-R promoter were introduced using the Stratagene Quick-Change method (Stratagene). Primer sequences for SF-1 - 35mut were $5^{\prime}$ CCCTTCCCGGCCCAAAATCCACTTGCTTGC3' and 5'GCAAGCAAGTGGATTTTGGGCCGGGAAGGG3, for SF-1 - 98mut 5'GTGGGATGACATTTATTCAAAATAATGATAACAATCTAG $3^{\prime}$ and $5^{\prime}$ GCTAGATTGTTATCATTATTTTGAATAAATGTCATCCCAC ${ }^{\prime}$ and for SF-1 - 209mut 5'CATTGGAAGTAATTTTGACTAGCTGAGCTC $3^{\prime}$ and $5^{\prime}$ GAGCTCAGCTAGTCAAAATTACTTCCATG3'. The full-length promoter construct in pGL3 served as a template for the PCR reaction (30 s at $95^{\circ} \mathrm{C}$ followed by $14-16$ cycles of $30 \mathrm{~s}$ at $95^{\circ} \mathrm{C}$, $1 \mathrm{~min}$ at $55^{\circ} \mathrm{C}$ and $12 \mathrm{~min}$ at $68^{\circ} \mathrm{C}$ ). The parental, methylated strand was digested with DpnI and $3 \mu \mathrm{l}$ of the digestion mixture were used to transform $\mathrm{DH} 5 \alpha$ cells. The resulting plasmids were sequenced to confirm the mutated sequence.

\section{Electrophoretic mobility shift assay (EMSA)}

Oligonucleotides containing wild-type or mutated SF-1binding sites -98 or -35 were annealed by boiling for $10 \mathrm{~min}$ and cooled down slowly to room temperature (RT). Annealed oligonucleotides were labeled with $\left[\alpha-{ }^{32} \mathrm{P}\right]$ ATP using T4 polynucleotide kinase (New England Biolabs, Schwalbach, Germany) at $37^{\circ} \mathrm{C}$ for $30 \mathrm{~min}$. Unincorporated nucleotides were removed by spin columns (Qiagen). For the protein-DNA interaction, $3 \mu \mathrm{l}$ of in vitro translated SF-1 (TNT quick kit; Promega) were combined with $4 \mu \mathrm{l} 5 \times$ EMSA binding buffer (100 mmol/l Tris-HCl, $400 \mathrm{mmol} / \mathrm{l} \mathrm{KCl,} 5 \mathrm{mmol} / \mathrm{l} \mathrm{EDTA}$, $0.5 \mathrm{mmol} / \mathrm{l}$ dithiothreitol, $0.5 \mathrm{mg} / \mathrm{ml} \mathrm{BSA}$ and $50 \%$ glycerol). For competition reactions 5, 10 and $15 \mathrm{pmol}$ unlabeled competitor oligonucleotide were added and the mixture was incubated for $20 \mathrm{~min}$ at RT. Subsequently, $3 \mu \mathrm{g}$ poly-dIdC (Sigma) and 20000 c.p.m. labeled oligonucleotide (roughly $0.01 \mathrm{pmol}$ ) were added and this mixture was incubated for another $20 \mathrm{~min}$ at RT. The reactions were electrophoresed on a $4 \%$ polyacrylamide gel at $150 \mathrm{~V}$ in $0.25 \times 22,5 \mathrm{mM}$ Trisborate, $0.5 \mathrm{mMEDTA}$ (TBE). Gels were dried and exposed to Kodak XAR film overnight.

\section{Results}

\section{ACTH-R and DAX-1 expression is negatively correlated in adrenocortical tumors}

ACTH-R gene expression is down-regulated or lost in a subset of adrenocortical tumors $(2,5)$. In order to investigate whether DAX-1 is involved in this effect, we investigated ACTH-R mRNA expression in 22 adrenocortical tumors and correlated it with DAX-1 expression (16). Although expression levels of both genes were variable in the tumor samples (Fig. 1A), an inverse correlation between ACTH-R and DAX-1 expression $(y=195.88-0.71883 x$ with $R=-0.47$, $P<0.02$ ) was found (Fig. 1B). Thus, these findings give indirect evidence that ACTH-R expression might be suppressed by DAX-1.

\section{DAX-1 inhibits basal and forskolin-induced ACTH-R promoter activity in vitro}

To further specify whether DAX-1 is directly involved in repression of ACTH-R gene expression, co-transfection of a DAX-1 expression plasmid with human and mouse full-length promoters were performed. DAX-1 reduced basal transcription of the hACTH-R and 
(A)
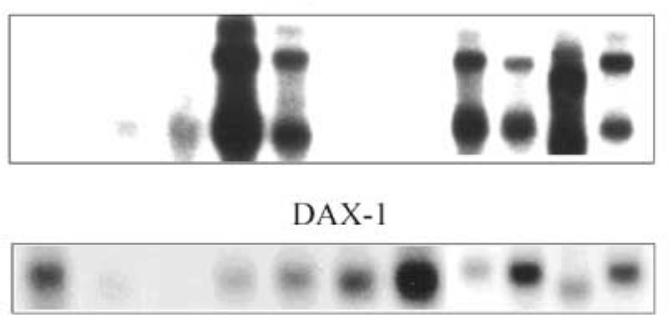

Actin

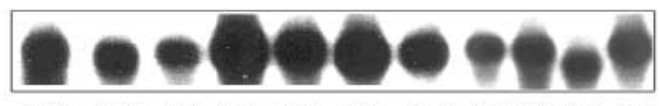

ACC ACC APA APA CPA NFA NFA APA NA APA NA

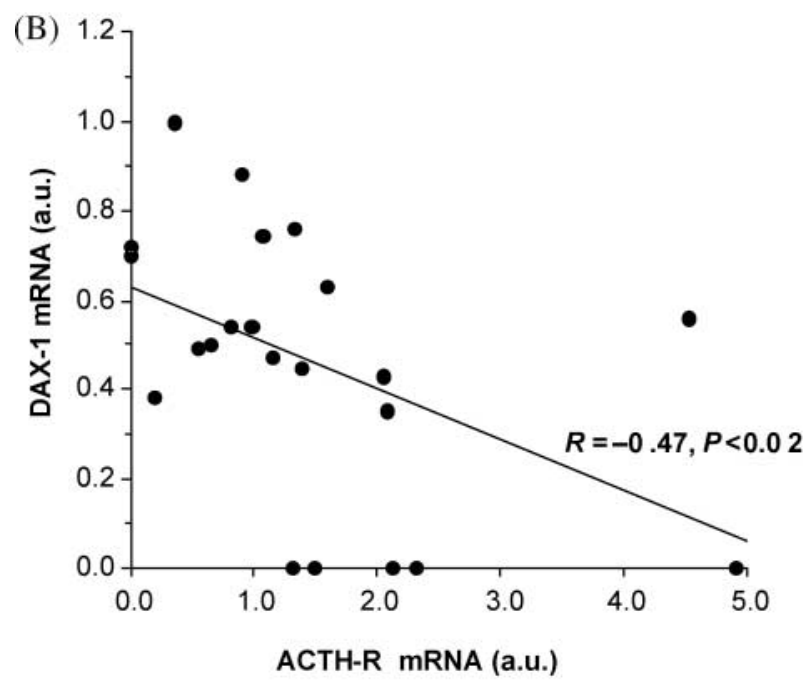

Figure 1 Northern blot for ACTH-R, DAX-1 and $\beta$-actin of adrenocortical tumors (A). NA: normal adrenal, ACC: adrenocortical carcinoma, APA: aldosterone-producing adenoma, CPA cortisolproducing adenoma, NFA: non-functioning adenoma. (B) Blot of ACTH-R and DAX1 expression of the 22 tumors investigated indicating a negative correlation between ACTH-R and DAX-1 expression. a.u., arbitrary units.

mACTH-R promoter by $76 \pm 5 \%$ (mean \pm s.E.M.) and $55 \pm 2 \%$ respectively (Fig. $2 \mathrm{~A}$ and $\mathrm{B}$ ). The respective forskolin-induced transcription was repressed by $84 \pm 4 \%$ (hACTH-R promoter) and 55 $2 \%$ (mACTH-R promoter), suggesting that DAX-1 inhibits basal and forskolin-stimulated mACTH-R and hACTH-R expression.

To further define the site of DAX-1 action in the hACTH-R promoter, 5'-truncated luciferase constructs extending from nucleotides $-783,-549,-293$, $-214,-112$ and -64 to nucleotide +40 were generated. Co-transfection of the constructs with a DAX-1 expression vector resulted in significant transcriptional inhibition of each of the constructs. The degree of DAX-1 inhibition decreased with the construct length and was strongest $(89 \%)$ in the full-length construct $p(-1017 /+40)$. However, luciferase activity of the constructs $-783,-549,-293,-214,-112$ and -64 was also reduced by $88,60,64,70,45$ and
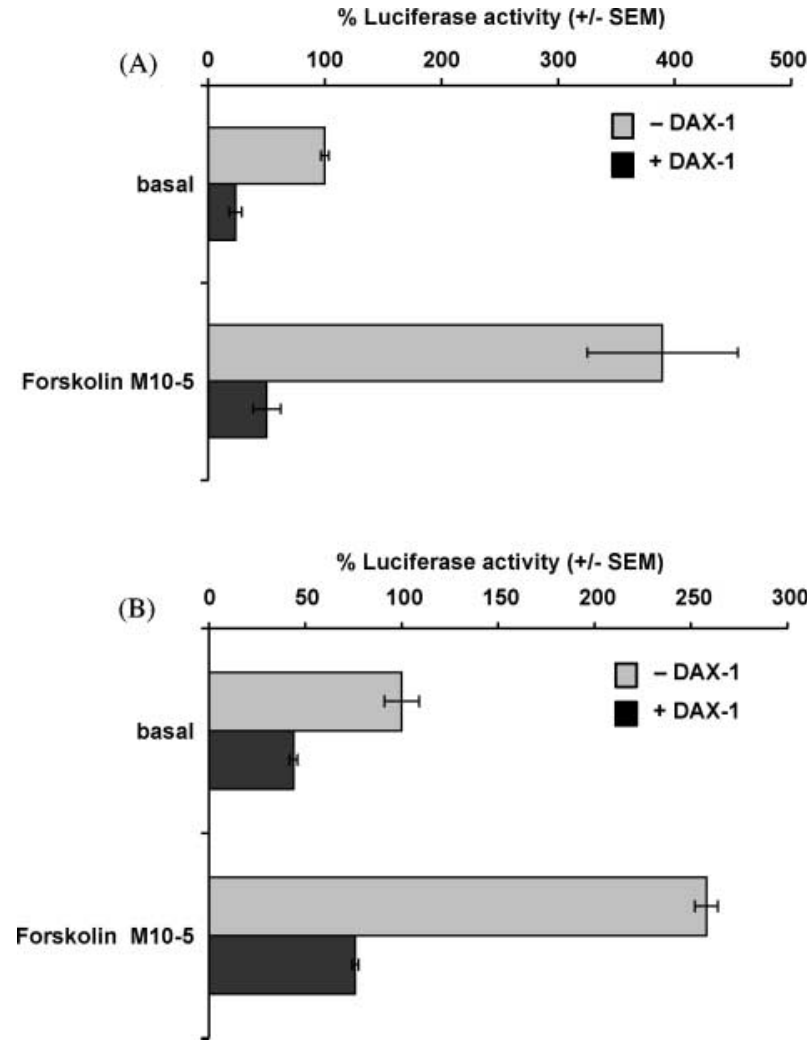

Figure 2 Inhibition of human (A) and mouse (B) ACTH-R promoter activity by DAX-1 (black bars) with and without forskolin stimulation suggesting DAX-1 induced repression of ACTH-R promoter activity. Luciferase activity of the full-length $(-1017 /+40)$ promoter construct was set to $100 \%$ and background activity of the empty vector pGL3 was subtracted.

$47 \%$ respectively (Fig. 3). Since all the investigated deletion constructs contain at least one SF-1-binding site, these findings are in line with the concept of SF-1 dependency of DAX-1 promoter repression.

\section{DAX-1 inhibition of ACTH-R promoter activity is dependent upon functional SF-1-binding sites}

In order to examine the role of SF-1 in DAX-1-mediated repression, we sequentially eliminated the three SF-1binding sites described in the hACTH-R promoter at positions -209 , -98 and -35 (20) by site-directed mutagenesis (19). The mutations were verified by sequencing and absence of SF-1 binding to the mutated DNA was shown by EMSA, where a upper non-specific band and specific, replaceable lower band were visible (Fig. 4A). Furthermore, transfection studies with the mutated hACTH-R promoter constructs and an SF-1 expression vector showed loss of SF-1-induced stimulation of promoter activity. Whereas wild-type promoter showed 3.4-fold stimulation by SF-1, this was reduced to 1.8-, 1.2-, 1.4- and 0.94-fold stimulation by mutation of the SF-1-binding sites at $-209,-98$ and $-35 \mathrm{bp}$ and all three binding sites respectively. 


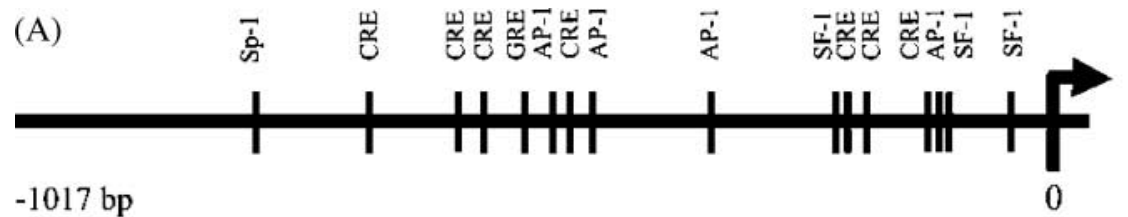

(B)

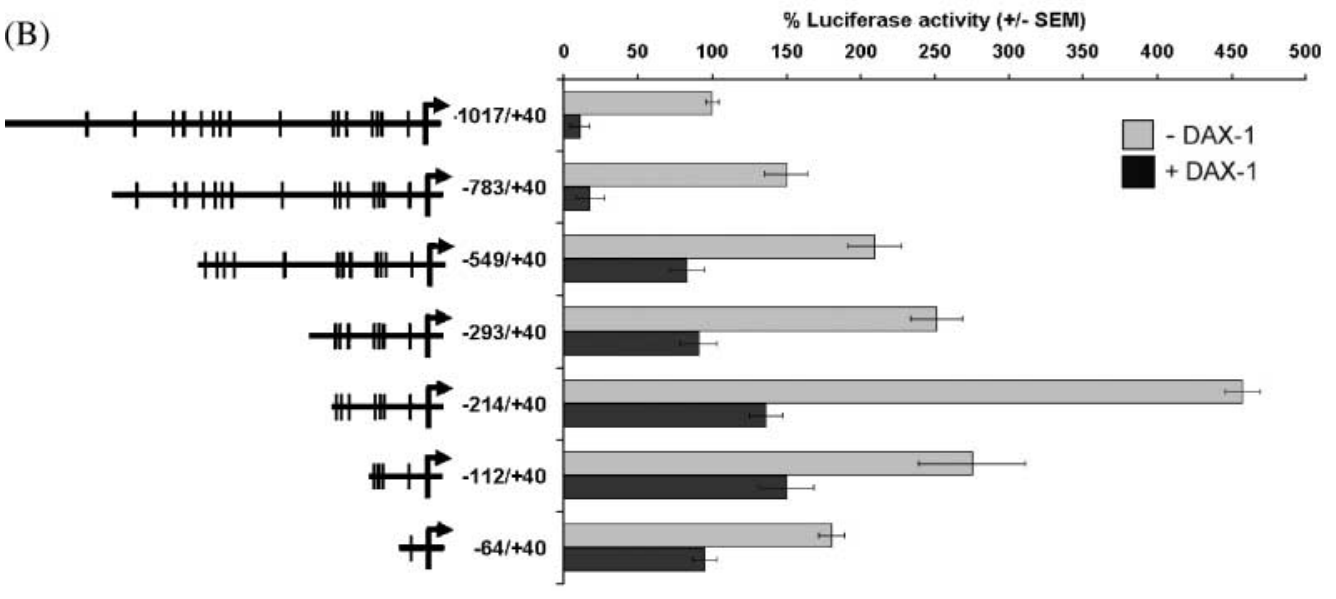

Figure $3(A)$ Scheme of the hACTH-R promoter and putative binding sites. (B) Relative luciferase activity of the human fulllength ACTH-R promoter and of $5^{\prime}$-deletion constructs with (black bars) and without DAX-1 (gray bars) indicating DAX-1induced repression of ACTH-R promoter activity in all deletion constructs. Full-length wild-type promoter was set to $100 \%$ and background activity of the empty vector pGL3 was subtracted in all experiments.
Co-transfection of the mutated constructs with DAX-1 reduced basal transcription of 209 mut by $27 \%$ and of 98 mut by $28 \%$, compared with $44 \%$ for the wild-type sequence. Moreover, DAX-1-dependent inhibition of ACTH-R promoter activity was completely abolished in the construct mutated at the -35 SF-1binding site (Fig. 4B). The triplicate mutant construct had a very low basal promoter activity of $9.5 \%$ as compared with that of the full-length promoter. As expected, co-transfection with the DAX-1 expression plasmid did not further inhibit ACTH-R promoter activity significantly. Taken together, these results indicate that DAX-1-induced suppression of ACTH-R expression requires functional SF-1-binding sites within the ACTH-R promoter, suggesting a direct and specific interaction between SF-1 and DAX-1 for ACTH-R transcriptional regulation.

\section{Discussion}

As we show herein, ACTH-R expression in adrenal tumors is negatively correlated with DAX-1 expression, providing indirect evidence that DAX-1, in addition to its known inhibitory effects on steroidogenic enzyme gene expression, might also inhibit ACTH-R promoter activity. In accord with this concept, it has been demonstrated that DAX-1 insufficiency in mice is associated with higher adrenal responsiveness to ACTH, presumably through increased expression of ACTH receptor and 21-hydroxylase (21). Thus, these findings point towards a further level of DAX-1-dependent repression of adrenal function. However, the exact molecular mechanisms of DAX-1-dependent suppression of ACTH-R gene transcription have not been defined.
We provide evidence that SF-1 and DAX-1 are functional antagonists for ACTH-R gene transcription in vitro and DAX-1-induced promoter inhibition requires functional SF-1-binding sites within the promoter region of the ACTH-R gene. DAX-1 inhibited the transcription of ACTH-R promoter luciferase constructs of both the hACTH-R and the mACTH-R promoter. In $5^{\prime}$-deletion constructs this inhibitory effect was still present with the shortest promoter construct $(-64 /+40)$, in which only one SF-1-binding site has been described (22). We therefore investigated the requirement of SF-1 function in DAX-1-dependent gene repression by creating promoter constructs with mutated SF-1-binding sites. As expected, baseline promoter activity was gradually decreased by elimination of the SF-1-binding sites, with mutation at position -209 or -98 having little effect. However, elimination of the -35 SF-1-binding site had the strongest effect on baseline gene transcription, and concomitant mutation of all three SF-1-binding sites further reduced ACTH-R gene transcription to $9.5 \%$ compared with the wild-type promoter. Likewise, co-transfection of a DAX-1 expression plasmid with the mutated promoter constructs at the -209 or -98 binding site revealed a reduced but still detectable inhibitory effect of DAX-1 on ACTH-R gene expression. In contrast, mutation of the binding site located next to the transcription start site at $-35 \mathrm{bp}$ abolished any significant DAX-1-dependent inhibitory effect on ACTH-R gene transcription. These results are in line with those reported for the CYP17 promoter, in which the inhibitory effect of DAX-1 on gene expression is reduced when less than three SF-1binding sites are present and is absent when only one SF-1-binding site is left (13). As for ACTH-R promoter 
(A) Probe: SF-1 -35 wt
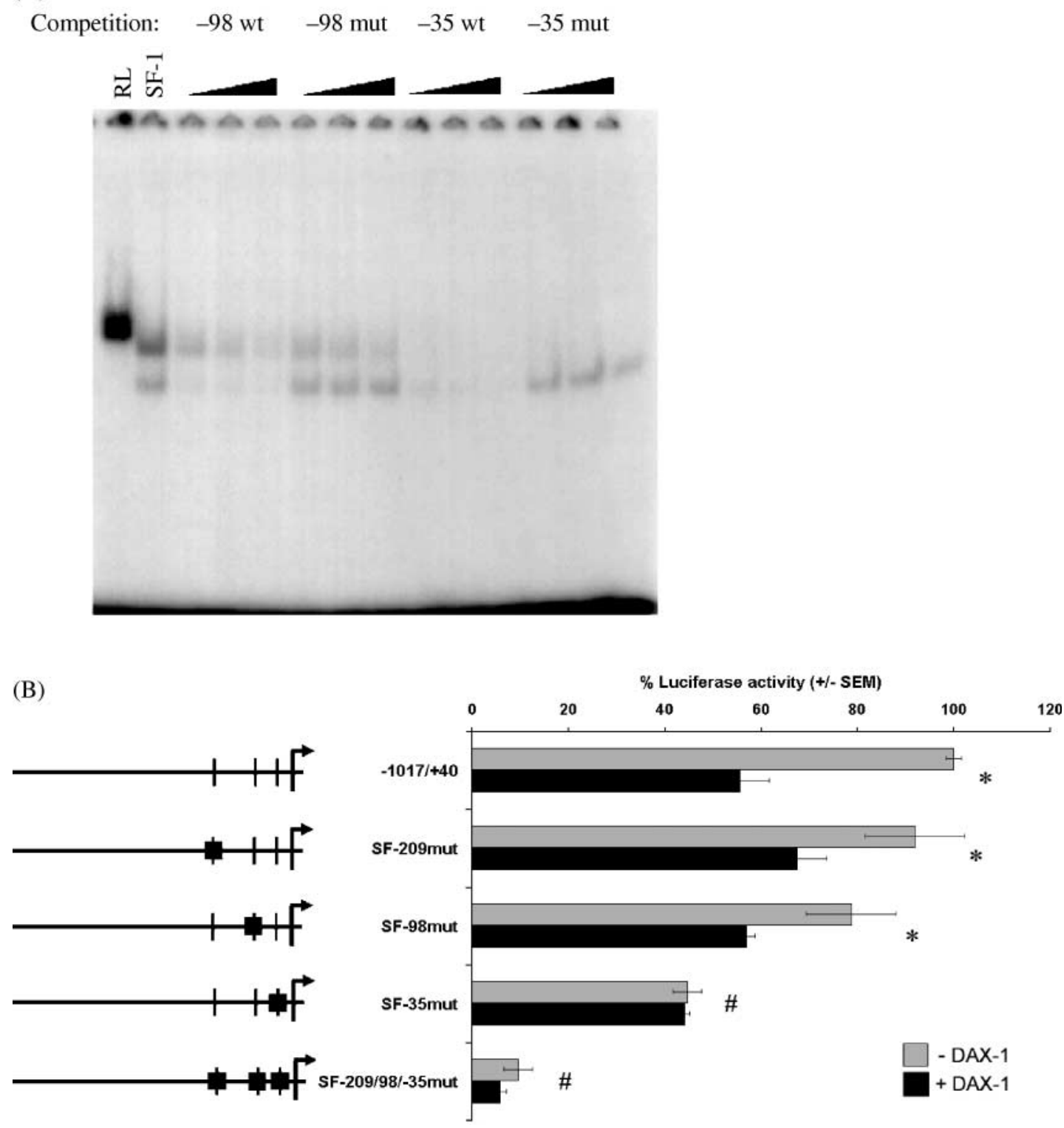

Figure 4 (A) EMSA, demonstrating loss of SF-1 binding to the mutated sequences. While the wild-type SF-1-binding site at position $-35 \mathrm{bp}$ after incubation with recombinant SF-1 results in an upper non-specific and a lower specific band, addition of increasing amounts of wild-type (wt) and mutated (mut) SF-1-binding sites at position -98 and -35 bp revealed competition only with the wildtype but not with the mutated oligonucleotides (RL: reticulocyte lysate). (B) Relative luciferase activity of the human full-length ACTH-R promoter of constructs mutated in the three SF-1-binding sites at position $-35,-98$ and $-209 \mathrm{bp}$ relative to the transcription start site. The reduction or loss of DAX-1-induced repression of luciferase activity in the mutated constructs indicates that DAX-1 inhibition of ACTH-R promoter activity is dependent upon functional SF-1-binding sites (for details see text). Full-length wild-type promoter was adjusted to $100 \%$ and background activity of the empty vector pGL3 was subtracted in all experiments. Gray bars: basal values, black bars: DAX-1. The mutated SF-1-binding sites are depicted as black boxes. *Statistical significance; \#no significant differences between DAX-1-transfected and control experiments.

activity, the -35 bp-located SF-1-binding site functionally seems to be the single most important one, as baseline promoter activity was significantly reduced and the inhibition by DAX-1 was absent in the corresponding mutated constructs. Although Naville et al. (3) reported mutation of the -35 SF-1-binding site resulting in the weakest reduction of basal promoter activity, these differences could be explained by the use of a $(-1017 /+40)$ full-length construct for mutational analyses in our studies instead of a truncated $(-263 /+22)$ construct in the latter. While using a shorter promoter fragment has the advantage of eliminating the influence of upstream promoter elements, one might regard a longer fragment as a construct 
better reflecting the situation in the endogenous gene promoter.

The regulation of the ACTH-R gene is unique in that it is up-regulated by its own ligand, ACTH (23). The hypothalamic-pituitary-adrenal (HPA) axis plays a pivotal role in physiological stress response and maintenance of metabolic homeostasis. Ligand-induced upregulation of ACTH-R expression may be an important adaptive process directed toward modulating adrenal responsiveness to ACTH. Whereas enhancement by ligand-induced up-regulation permits a more efficient and rapid glucocorticoid response in the adrenal cortex, negative feedback regulation by glucocorticoids in the hypothalamus and pituitary inhibits ACTH secretion and allows a balanced adrenal response to stress. The exact molecular mechanisms that transduce activation of the ACTH-R to induce target gene expression remain the subject of current investigation and might include direct activation of cAMP response elements as well as post-transcriptional modification of transcription factors including SF-1 (24). As shown herein, ACTH-R promoter activation by forskolin - a compound which increases intracellular cAMP production and hence activates the protein kinase A (PKA) pathway - can be significantly reduced by DAX-1 for both the human and mouse promoter constructs. These findings indicate that PKA pathway-dependent gene activation can be modulated by DAX-1. In line with this concept, up-regulation of adrenal ACTH-R gene transcription in mice following chronic stimulation of the HPA axis is further increased in the context of DAX-1 insufficiency as compared with wild-type animals (21).

We cannot rule out the possibility that mechanisms of DAX-1 repression on ACTH-R promoter activity other than direct interaction with SF-1 may exist. These could include SF-1-independent binding of DAX-1 to DNA hairpin structures, repression of SF-1 gene expression by DAX-1 or DAX-1-dependent induction of other transcriptional co-repressors. However, since sequence analysis of the hACTH-R promoter revealed no DNA hairpin structures close to the SF-1binding sites and only single point mutations were introduced specifically at the SF-1-binding sites, SF-1independent DNA binding of DAX-1 seems unlikely to play a major role for ACTH-R gene expression regulation. Moreover, expression studies in human adrenal tumor samples (16) and mouse models of DAX-1 deficiency (21) demonstrate unaltered SF-1 mRNA or protein levels independently of DAX-1 expression levels. These findings suggest that DAX-1 does not transcriptionally or translationally regulate SF-1 in the adult adrenal cortex and argue against a DAX-1-dependent repression of SF-1 gene expression as the cause of the observed inhibitory effects of DAX-1 on ACTH-R promoter activity. Moreover, we cannot exclude the possibility that trans-activating transcription factors other than SF-1 might be suppressed by DAX-1, thus contributing to further inhibition of ACTH-R promoter activity. However, since DAX-1 was not able to suppress luciferase activity at the construct specifically mutated in the SF-1-binding site at $-35 \mathrm{bp}$, this finding suggests that there is indeed a close and functionally specific interaction between SF-1 and DAX-1 for ACTH-R transcriptional regulation.

Adrenal incidentalomas are the most common neoplasia of the adrenal cortex with a prevalence of up to $6 \%$ in the otherwise healthy population (25). Although the vast majority of cases represent endocrine-inactive adrenal adenomas and although there is only a minimal chance for the presence of an adrenal carcinoma in patients with small adrenal lesions, in individual patients it can prove difficult to distinguish between these benign adenomas and adrenal carcinomas even with histopathological and immunohistochemical methods. Thus, clinical or molecular markers that would help to distinguish benign from malign adrenal tumors would be welcome. Our data indicate that lower responsiveness to $\mathrm{ACTH}$ in vivo as well as lower expression of ACTH-R and higher expression of DAX-1 might give additional insights in the pathogenesis of adrenal tumors in this group of patients. However, more detailed expression profiles from adrenocortical carcinomas at different stages are needed before clinical applications could be considered.

\section{Acknowledgements}

This work was supported by a grant from the Deutsche Forschungsgemeinschaft (DFG) to M R (Re 752 11/1).

\section{References}

1 Mountjoy KG, Robbins LS, Mortrud MT \& Cone RD. The cloning of a family of genes that encode the melanocortin receptors. Science $19922571248-1251$.

2 Reincke M, Beuschlein F, Latronico AC, Arlt W, Chrousos GP \& Allolio B. Expression of adrenocorticotrophic hormone receptor mRNA in human adrenocortical neoplasms: correlation with P450scc expression. Clinical Endocrinology 199746 619-626.

3 Naville D, Penhoat A, Durand P \& Begeot M. Three steroidogenic factor-1 binding elements are required for constitutive and cAMPregulated expression of the human adrenocorticotropin receptor gene. Biochemical and Biophysical Research Communications 1999 $25528-33$.

4 Sarkar D, Kambe F, Hayashi Y, Ohmori S, Funahashi H \& Seo H. Involvement of AP-1 and steroidogenic factor (SF)-1 in the cAMPdependent induction of human adrenocorticotropic hormone receptor (ACTHR) promoter. Endocrine Journal 200047 63-75.

5 Reincke M, Beuschlein F, Menig G, Hofmockel G, Arlt W, Lehmann R, Karl M \& Allolio B. Localization and expression of adrenocorticotropic hormone receptor mRNA in normal and neoplastic human adrenal cortex. Journal of Endocrinology 1998156 415-423.

6 Reincke M, Mora P, Beuschlein F, Arlt W, Chrousos GP \& Allolio B. Deletion of the adrenocorticotropin receptor gene in human adrenocortical tumors: implications for tumorigenesis. Journal of Clinical Endocrinology and Metabolism 199782 3054-3058.

7 Parker KL \& Schimmer BP. Steroidogenic factor 1: a key determinant of endocrine development and function. Endocrine Reviews $199718361-377$. 
8 Zazopoulos E, Lalli E, Stocco DM \& Sassone-Corsi P. DNA binding and transcriptional repression by DAX-1 blocks steroidogenesis. Nature $1997390311-315$.

9 Lalli E, Melner MH, Stocco DM \& Sassone-Corsi P. DAX-1 blocks steroid production at multiple levels. Endocrinology $1998 \mathbf{1 3 9}$ $4237-4243$

10 Lalli E, Ohe K, Hindelang C \& Sassone-Corsi P. Orphan receptor DAX-1 is a shuttling RNA binding protein associated with polyribosomes via mRNA. Molecular and Cellular Biology 200020 4910-4921.

$11 \mathrm{Yu} \mathrm{RN}$, Ito M \& Jameson JL. The murine Dax-1 promoter is stimulated by SF-1 (steroidogenic factor-1) and inhibited by COUP-TF (chicken ovalbumin upstream promoter-transcription factor) via a composite nuclear receptor-regulatory element. Molecular Endocrinology 199812 1010-1022.

12 Kawabe K, Shikayama T, Tsuboi H, Oka S, Oba K, Yanase T, Nawata H \& Morohashi K. Dax-1 as one of the target genes of Ad4BP/SF-1. Molecular Endocrinology 199913 1267-1284.

13 Hanley NA, Rainey WE, Wilson DI, Ball SG \& Parker KL. Expression profiles of SF-1, DAX1, and CYP17 in the human fetal adrenal gland: potential interactions in gene regulation. Molecular Endocrinology 200115 57-68.

14 Nachtigal MW, Hirokawa Y, Enyeart-VanHouten DL, Flanagan JN, Hammer GD \& Ingraham HA. Wilms' tumor 1 and Dax-1 modulate the orphan nuclear receptor SF-1 in sex-specific gene expression. Cell 199893 445-454.

15 Lalli E, Bardoni B, Zazopoulos E, Wurtz JM, Strom TM, Moras D \& Sassone-Corsi P. A transcriptional silencing domain in DAX-1 whose mutation causes adrenal hypoplasia congenita. Molecular Endocrinology 199711 1950-1960.

16 Reincke M, Beuschlein F, Lalli E, Arlt W, Vay S, Sassone-Corsi P \& Allolio B. DAX-1 expression in human adrenocortical neoplasms: implications for steroidogenesis. Journal of Clinical Endocrinology and Metabolism $1998 \mathbf{8 3} 2597-2600$.

17 Zanaria E, Muscatelli F, Bardoni B, Strom TM, Guioli S, Guo W, Lalli E, Moser C, Walker AP, McCabe ER, Meitinger T, Monaco AP, Sassone-Corsi P \& Camerino G. An unusual member of the nuclear hormone receptor superfamily responsible for X-linked adrenal hypoplasia congenita. Nature 1994372 635-641.

18 Lynch JP, Lala DS, Peluso JJ, Luo W, Parker KL \& White BA. Steroidogenic factor 1 , an orphan nuclear receptor, regulates the expression of the rat aromatase gene in gonadal tissues. Molecular Endocrinology $19937776-786$.

19 Marchal R, Naville D, Durand P, Begeot M \& Penhoat A. A steroidogenic factor-1 binding element is essential for basal human ACTH receptor gene transcription. Biochemical and Biophysical Research Communications 1998247 28-32.

20 Naville D, Penhoat A, Marchal R, Durand P \& Begeot M. SF-1 and the transcriptional regulation of the human ACTH receptor gene. Endocrine Research $199824391-395$.

21 Babu PS, Bavers DL, Beuschlein F, Shah S, Jeffs B, Jameson JL \& Hammer GD. Interaction between Dax-1 and steroidogenic factor-1 in vivo: increased adrenal responsiveness to ACTH in the absence of Dax-1. Endocrinology 2002143 665-673.

22 Naville D, Jaillard C, Barjhoux L, Durand P \& Begeot M. Genomic structure and promoter characterization of the human ACTH receptor gene. Biochemical and Biophysical Research Communications $19972307-12$.

23 Mountjoy KG, Bird IM, Rainey WE \& Cone RD. ACTH induces upregulation of ACTH receptor mRNA in mouse and human adrenocortical cell lines. Molecular and Cellular Endocrinology 1994 99 R $17-\mathrm{R} 20$.

24 Hammer GD, Krylova I, Zhang Y, Darimont BD, Simpson K, Weigel NL \& Ingraham HA. Phosphorylation of the nuclear receptor SF-1 modulates cofactor recruitment: integration of hormone signaling in reproduction and stress. Molecular Cell 19993 $521-526$

25 Reincke M, Beuschlein F, Slawik M \& Borm K. Molecular adrenocortical tumourigenesis. European Journal of Clinical Investigation $20003063-68$.

Received 23 September 2004

Accepted 11 February 2005 